\title{
Rapid Implementation of Remote Online Patient Portal Activation in a Children's Hospital during the COVID-19 Pandemic
}

\author{
Kim T. Vuong ${ }^{1,2}$ Tiranun Rungvivatjarus ${ }^{1,2}$ Isabel P. Garcia ${ }^{1} \quad$ Cindy Lewis $^{1} \quad$ Lisa Miller $^{1}$ \\ Cynthia L. Kuelbs ${ }^{1,2}$
}

${ }^{1}$ Department of Pediatrics, Rady Children's Hospital, San Diego, California, United States

${ }^{2}$ Department of Pediatrics, University of California, San Diego, San Diego, California, United States

Address for correspondence Kim T. Vuong, MD, MPH, Department of Pediatric Residency, 3020 Children's Way, MC 5124, San Diego, CA 92123, United Sates (e-mail: kivuong@health.ucsd.edu).

ACI Open 2021;5:e17-e26.

\begin{abstract}
Keywords

- patient portal

- telemedicine

- pediatrics

- patient engagement

- patient provider communication

Background Patient portal access allows more patient engagement, yet portal usage is not universal. Telemedicine visits became critical when the coronavirus disease 2019 (COVID-19) pandemic limited in-office visits. Given physical distancing requirements and telemedicine platform integrated into the patient portal, our institution needed to rapidly enroll patients and their proxies remotely, including hospitalized patients, to minimize care disruptions.

Objectives The study aimed to describe the rapid implementation of remote patient portal activation in a pediatric integrated delivery network during the COVID-19 pandemic. Additionally, to increase the percentage of patients with active portal status upon discharge by $15 \%$ (absolute change) across inpatient units within 3 months.

Methods A multidisciplinary taskforce utilized quality improvement tools to identify barriers to successful inpatient patient portal activation followed by rapid intervention cycles to effect change. Weekly progress was monitored on a portal activation dashboard.

Results We created clinical decision support tools and training within 2 weeks. Active patient portal status upon hospital discharge increased from 44 to $66 \%$ within 12 weeks. Online patient portal conversion within 7 days after hospital discharge increased from 6.0 to $24 \%$. Offer rate increased from 73 to $85 \%$ across all inpatient units.

Conclusion We rapidly and effectively implemented a remote proxy portal activation process in the inpatient setting. Remote activation, training, clinical decision support, multidisciplinary involvement, and emphasis on the portal as integral to ongoing care allowed us to dramatically increase portal conversion. This experience may be informative for pediatric hospitals aiming to increase proxy portal enrollment and adult hospitals with medical decision-making proxies.
\end{abstract}

received

June 27, 2020

accepted after revision

December 28, 2020
DOI https://doi.org/

10.1055/s-0041-1729983.

ISSN 2566-9346. (c) 2021. The Author(s).

This is an open access article published by Thieme under the terms of the Creative Commons Attribution License, permitting unrestricted use, distribution, and reproduction so long as the original work is properly cited. (https://creativecommons.org/licenses/by/4.0/)

Georg Thieme Verlag KG, Rüdigerstraße 14, 70469 Stuttgart, Germany 


\section{Introduction}

On December 31st, 2019, China reported several cases of pneumonia of unknown etiology in Wuhan soon identified as a novel coronavirus, severe acute respiratory syndrome coronavirus 2 (SARS-CoV-2). ${ }^{1,2}$ The virus spread throughout the Hubei province and then throughout the world. In the United States, the first case of SARS-CoV-2 was reported in late January, followed by a cluster of cases in Washington..$^{3-7}$ With cases numbering $>100,000$, the World Health Organization declared the outbreak a pandemic on March 11, $2020 .^{8}$ The United States declared a national emergency on March 13, 2020 and shortly thereafter implemented physical distancing guidelines. ${ }^{9,10}$

In a pandemic, health care systems need to prepare for sudden and frequent changes in patient care and disruption of usual workflows. With the COVID pandemic, many health care systems pivoted to telemedicine to minimize unnecessary exposures to the patient and treatment team while still allowing for ongoing care. We were no exception and identified several barriers to rapid expansion of ambulatory telemedicine. First, our institutional telemedicine visits occur via Zoom (Zoom Video Communications Inc., San Jose, California, United States) for the video component, which is integrated into our online patient portal, MyChart (Epic System Corporation, Verona, Wisconsin, United States). This allows the provider to easily visualize the patient and navigate the electronic medical record (EMR) simultaneously, more closely replicating an in-person visit. To fully utilize telemedicine, patients are required to have an active patient portal account. Therefore, we needed to rapidly activate patient portal accounts which led to the second barrier. Given that most of our patient portal access is through parent/caregiver or "proxy" access, our consent and activation process requires proof of identity. The physical distancing requirements stipulated the need to activate remotely, whereas previously activation required in-person sign up. The last barrier was cultural. As we do not have an inpatient portal, portal access had been considered an ambulatory responsibility with usage rates around 50\% among ambulatory patients; inpatient care areas were not focused on granting portal access.

As general pediatrician and subspecialty clinic appointments rapidly converted to telemedicine visits, hospitalized patients were faced with the inability to complete posthospitalization follow-up without active patient portal access. This could lead to poor transition of care and high risk for readmission due to the lack of follow-up. Given the physical distancing requirements imposed by the pandemic, we needed to ensure that patients could be seen postdischarge via telemedicine, if deemed appropriate. Activating the portal prior to discharge would facilitate telemedicine for follow-up visits; however, only $44 \%$ of our inpatients had an active portal account upon hospital discharge. Our patient portal conversion rate within 7 days of hospital discharge was $6 \%$, and we were offering portal activation to $73 \%$ of admitted patients. A MyChart activation task force was formed to better understand the problem, formulate solu- tions, and implement processes to improve the percentage of patients with active portal accounts.

\section{Objectives}

Our aims were to rapidly implement remote patient portal activation in a quaternary care, academic children's hospital, and integrated delivery network during the initial phase of the COVID-19 pandemic and to increase the percentage of active patient portal status upon discharge by $15 \%$ (absolute change) across all inpatient units within 3 months.

\section{Methods}

\section{Context}

We are a pediatric integrated delivery network comprised of primary care and specialty practices as well as a quaternary care 551-bed hospital located in Southern California. We serve over 260,000 children with approximately 20,000 annual admissions. Our institution is the primary inpatient pediatric training site for residents and fellows from our affiliated school of medicine and five other local training programs. In 2009, we transitioned from a paper-based health record to an electronic health records system (Epic, Epic Systems Corporation, Verona, Wisconsin, United States) that includes computerized provider order entry with clinical decision support. Inpatient units transitioned in 2011. We have had a patient portal since 2011 .

\section{Planning the Intervention}

Starting in March 2020, a multidisciplinary task force was created by the chief medical information officer and a hospitalist/physician informaticist, who co-led a team of attending physicians, resident physicians, hospital unit clerks (HUC), medical and surgical unit managers, patient account representatives (PARs), and registered nurses (RNs). The task force met on a weekly basis to define the online patient access enrollment process, review data, assess barriers, and implement interventions. Process maps and fishbone diagrams ( - Fig. 1) were used to identify MyChart activation workflows and barriers. Prior to the physical distancing required by the pandemic, online patient portal enrollment occurred passively on hospital admission. For patients on standard isolation precautions, caregivers would stop at the HUC station and were given a MyChart proxy paper form. Caregivers would return the completed paper form to the HUC, and an activation code was then emailed to the caregivers. The caregivers would click on the activation code embedded hyperlink which then activated their child's MyChart account. As caregivers of patients on contact/droplet precautions do not stop at the HUC station upon arrival on the unit to minimize exposure, they were not given the MyChart proxy paper form. If portal access was requested by the caregiver, caregivers were instructed to leave the patient's room and go to the HUC station to obtain the MyChart proxy form and follow the same enrollment process as described above. 


\section{Causes of Low MyChart Activation}

Equipment Procedures Patients

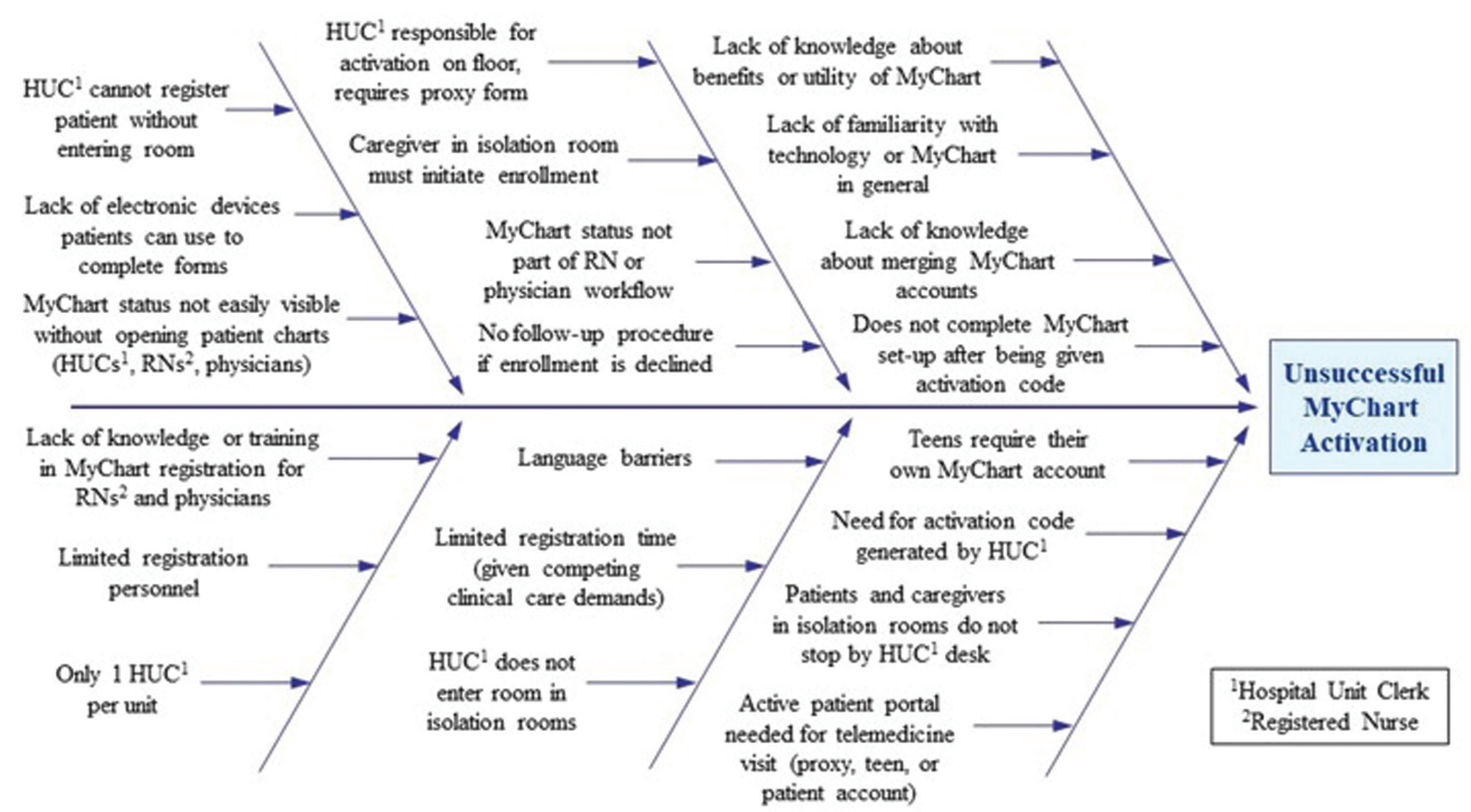

Staff

Environment

Policies

Fig. 1 Fishbone diagrams to identify barriers to the MyChart activation workflow.

\section{Measures}

Our MyChart statuses are categorized into six types: activated, inactivated, pending activation, patient declined, activation code generated but disabled, and nonstandard MyChart status. The definition of each status is listed in - Table 1. A blank portal status, without any one of the six types, was defined as a failure to offer MyChart enrollment. Portal conversion within 7 days was defined as successful when a patient chart with blank or any nonactive MyChart status converted to "activated" within 7 days from the time the activation code was generated. The primary outcome measure was the percentage of admitted patients with active
MyChart status upon discharge from the hospital. Our secondary outcome measures were the percentage of patients offered enrollment and the percentage of patients successfully converted to active status within 7 days.

\section{Data Collection and Analysis}

Baseline data from October 2019 to the end of March 2020 were collected retrospectively via Epic Reporting Workbench. Qualitative data were collected via fishbone diagram and process map as noted previously. Weekly percentage data were collected prospectively via Epic Reporting Workbench. We used statistical process control charts to examine

Table 1 MyChart statuses and definitions

\begin{tabular}{|l|l|}
\hline MyChart status & Definition \\
\hline Activated & These patients have an active MyChart account \\
\hline Inactivated & $\begin{array}{l}\text { These patients had an active MyChart account at some point, but the account has been } \\
\text { inactivated. Administrators can inactivate accounts manually, and accounts can be inactivated } \\
\text { automatically based on a patient action such as successive failed login attempts }\end{array}$ \\
\hline Pending activation & $\begin{array}{l}\text { A staff member generated MyChart activation codes for these patients, but the patients have not } \\
\text { used the code to log in to MyChart and activate an account }\end{array}$ \\
\hline $\begin{array}{l}\text { Nonstandard } \\
\text { MyChart status }\end{array}$ & $\begin{array}{l}\text { A nonstandard status might occur if your organization uses Interconnect Web services for } \\
\text { MyChart. For example, Interconnect might create a MyChart patient record on the fly to } \\
\text { complete a workflow and then set that patient's MyChart status to unknown }\end{array}$ \\
\hline Patient declined & $\begin{array}{l}\text { These patients declined a MyChart account when they were asked whether they wanted to sign } \\
\text { up }\end{array}$ \\
\hline
\end{tabular}


the impact of quality improvement interventions. Control limits were set at \pm 3 standard deviations per conventional standard and adjusted during the postintervention period based on special cause variation. ${ }^{11,12}$

\section{Interventions}

Members of the task force collected information on barriers to patient portal enrollment via phone interviews with families, HUCs, and PARs. Information collected was used to complete a fishbone diagram and process map. Barriers to successful online patient portal enrollment for admitted patients included lack of efficient enrollment process for patients on contact/droplet precautions, lack of engagement by the medical team, nonexistent follow-up procedures when enrollment was declined, caregiver's lack of knowledge regarding online patient portal benefits, and caregiver's unfamiliarity with technology. Major barriers identified were addressed with specific interventions as summarized in - Table 2. - Table 2 includes both implemented interventions and planned future interventions.

With the onset of the pandemic and need for rapid transition to telemedicine, we quickly developed a remote portal enrollment process which allowed remote electronic identity verification and phone enrollment. Caregivers are granted partial access to their child's portal where they can electronically upload an official identification document for verification. Once registration staff verifies the identification document, caregivers are then provided with full proxy access and the activation code is emailed to caregivers. At the start of our quality improvement initiative, the remote online enrollment process occurring in the ambulatory settings had yet to be implemented on the inpatient units.

To address the barriers identified and increase our enrollment, we implemented multiple cycles of interventions spanning from April 2, 2020 to May 8, 2020. Our first intervention was focused on rapid cycle training of the remote MyChart enrollment workflow. HUCs across all inpatient units at the main hospital and satellite units (medical, surgical, hematology/oncology, critical care, neonatology, and cardiac intensive care units) were the first to receive training, mostly via emails and pictorial tip sheets. Due to physical distancing guidelines, in-person training was not possible and live video conferencing was limited as few HUCs work at any given time. Thus, to enhance training for HUCs,

Table 2 Linkage between identified barriers and selected interventions. Major barriers identified with specific interventions, including both implemented interventions and planned future interventions

\begin{tabular}{|c|c|}
\hline Barriers & Interventions \\
\hline $\begin{array}{l}\text { Lack of knowledge and training in patient portal registration for nurses or } \\
\text { physicians Lack of staff knowledge about the remote patient portal } \\
\text { activation process }\end{array}$ & \multirow[t]{2}{*}{$\begin{array}{l}\text { Rapid cycle training of HUC, physicians, and } \\
\text { nurses }\end{array}$} \\
\hline - HUC previously used proxy paper form for patient portal activation & \\
\hline $\begin{array}{l}\text { Lack of caregiver knowledge about patient portal benefits and unfa- } \\
\text { miliarity with technology }\end{array}$ & \multirow{2}{*}{$\begin{array}{l}\text { Caregiver/patient education included in } \\
\text { "Welcome Packet" on hospital admission } \\
\text { Patient portal education video being made in } \\
\text { English, Spanish, and Arabic }{ }^{\text {a }}\end{array}$} \\
\hline $\begin{array}{l}\text { - Lack of familiarity with patient portal application or technology in } \\
\text { general } \\
\text { - Lack of knowledge about merging MyChart accounts } \\
\text { - Caregiver does not complete patient portal activation even after } \\
\text { activation code is given }\end{array}$ & \\
\hline $\begin{array}{l}\text { Lack of efficient enrollment process for patients on contact/droplet } \\
\text { precautions. Nonexistent follow-up procedures when enrollment was } \\
\text { declined }\end{array}$ & \multirow[t]{2}{*}{ Standardized portal enrollment workflow } \\
\hline $\begin{array}{l}\text { - Patients and caregivers in contact/droplet rooms do not stop by HUC } \\
\text { desk } \\
\text { - Caregivers in contact/droplet room must initiate enrollment } \\
\text { - HUC does not enter room in contact/droplet rooms } \\
\text { - HUC cannot register patient without entering room }\end{array}$ & \\
\hline Lack of engagement by the hospital team & \multirow[t]{2}{*}{ Improved visibility of portal status } \\
\hline $\begin{array}{l}\text { - Patient portal activation is not part of nurse or physician workflow } \\
\text { - MyChart status not easily visible without opening patient charts } \\
\text { (HUCs, nurses, and physicians) }\end{array}$ & \\
\hline Lack of equipment or resource & \multirow{2}{*}{$\begin{array}{l}\text { Request to hospital leadership for additional } \\
\text { personnel that would be dedicated to patient } \\
\text { portal activation } \\
\text { Request for bedside tablets to be available for } \\
\text { patient portal activation }\end{array}$} \\
\hline $\begin{array}{l}\text { - Lack of electronic devices patients can use to complete forms } \\
\text { - Limited HUC and registration personnel } \\
\text { - Limited registration time (given competing clinical care demands) }\end{array}$ & \\
\hline Language barrier & $\begin{array}{l}\text { Dedicate analyst resources to non-English, } \\
\text { non-Spanish patient portal application build }\end{array}$ \\
\hline
\end{tabular}


task force members used phone calls to individual HUCs to help reinforce the change in workflow and to identify barriers to the new workflow over a course of 1 week. Multiple rapid cycles of training were expanded to include PARs, nurses, nurse educators, parent liaisons, and physicians. Training for resident physicians occurred via tip sheet, emails, and video conferences. Given the lack of inpatient medical team engagement in MyChart enrollment previously, training for inpatient physicians and nurses was expanded to also include the various functionalities of MyChart and commonly asked questions.

To increase caregiver awareness of online patient portal benefits, our second intervention focused on patient education. Educational materials on MyChart functionality including telehealth visit capability and access to COVID-19 test results posthospitalization were added to all "Welcome Packets" given to families upon admission. Bedside nurses were responsible for reviewing the "Welcome Packets" with families and for notifying the parent liaison if caregivers appeared hesitant to enroll. The parent liaison would then educate caregivers by phone on portal benefits from a patient perspective. To further engage the medical team in the process, the task force also created a new HUC-driven protocol which provided HUCs with steps to notify the primary treatment team on both medical and surgical units if a caregiver declined enrollment. When notified by HUCs, physicians are expected to discuss with caregivers why they declined to enroll and address any questions or concerns.

Our third intervention aimed to standardize portal enrollment across all inpatient units through a HUC-driven workflow. With remote portal enrollment, HUCs were able to enroll patients with contact/droplet precautions the same way they enroll standard isolation precaution patients without an increase in exposure risk. HUCs were to identify patients with a blank or inactivated MyChart status in Epic daily and call families to offer MyChart enrollment over the phone. A barrier quickly identified was that the HUC's unit manager view (default view and workspace in the EMR for HUCs) did not include patients' MyChart statuses, making it very difficult for HUCs to identify patients with a blank or inactivated MyChart status. This led to our final intervention.

Our fourth intervention was to enhance the HUC's and clinical staff's ability to quickly identify MyChart status. The HUC Unit Manager view was modified to highlight patient records with a blank or inactivated MyChart status, allowing HUCs to identify them easily (-Fig. 2). A patient list column was also created for physicians and nurses with different colors corresponding to various MyChart statuses, allowing clinicians to identify their patient's MyChart status from their daily patient lists (-Fig. 3). Visibility of portal status allows HUCs and medical providers to not only focus their enrollment efforts, but also to cohesively reinforce the importance of enrollment to families. A MyChart Activation Epic Dashboard was created for unit leadership and the task force to monitor progress on a weekly basis. The dashboard tracked changes in MyChart activation status within a 7-day period, offer rate, and the total percentage of active MyChart status.

\section{Ethical Consideration}

The project was reviewed by the local institutional review board and was deemed not to be human subjects research. Quality improvement institutional review was obtained and granted.

\section{Results}

The new remote online patient portal enrollment training was completed on all inpatient units within 2 weeks from task force creation. Clinical decision support tools (unit manager view and MyChart status Epic column) were also created within

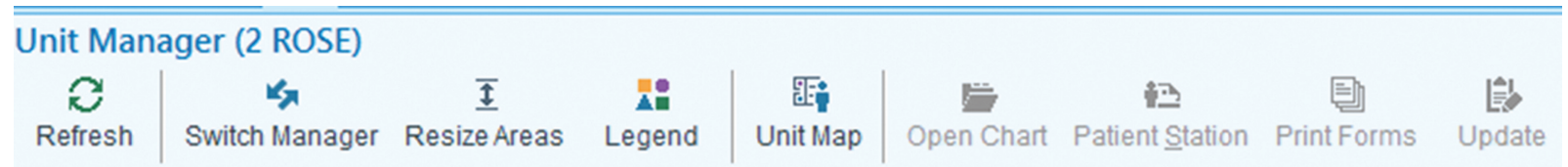

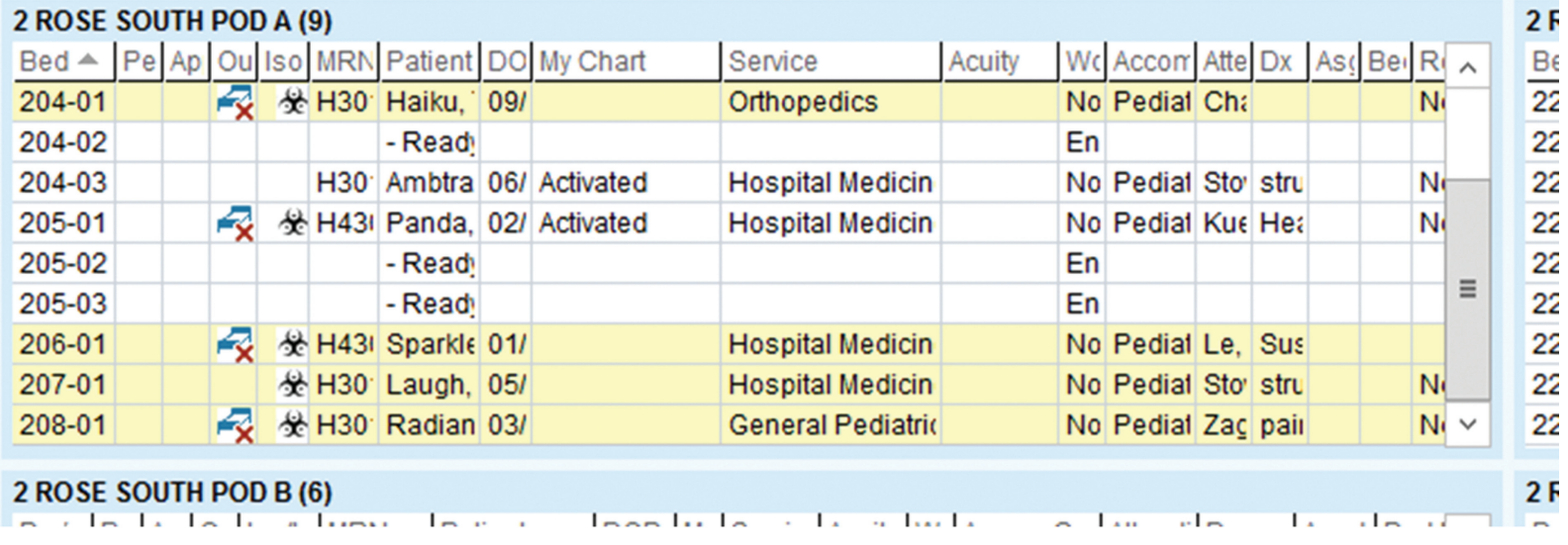

(C) 2020 Epic Systems Corporation. Used with permission.

Fig. 2 Hospital unit clerk (HUC) unit manager view modified to highlight patient records with a blank or inactivated MyChart status (highlighted in yellow). 


\begin{tabular}{|ll}
\hline Unit/Room/Bed & MyChart \\
\hline 2ROSE-201-01 & Activated \\
\hline 2ROSE-202-01 & Activated \\
\hline 2ROSE-203-01 & $\begin{array}{l}\text { Patient } \\
\text { Declined }\end{array}$ \\
\hline 2ROSE-205-01 & $\begin{array}{l}\text { Pending } \\
\text { Activation }\end{array}$ \\
\hline 2ROSE-206-01 & Activated \\
\hline 2ROSE-208-01 & $\begin{array}{l}\text { Patient } \\
\text { Declined }\end{array}$ \\
\hline 2ROSE-210-01 & Activated \\
\hline 2ROSE-213-01 & Activated \\
\hline 2ROSE-215-01 & - \\
\hline 2ROSE-216-01 & - \\
\hline @2020 Epic Systems Corporation. Used with permission.
\end{tabular}

Fig. 3 Patient list column in Epic with different colors corresponding to various MyChart statuses.

2 weeks. All admitted patients between October 2, 2019 and May 31, 2020 were included to track overall online patient portal activation percentages. Basic demographic data of the active and inactive MyChart patient population during the last week of our study was summarized in - Table 3. Using the Epic EMR MyChart Activation Dashboard, we tracked the percentage of hospitalized patients in each unit whose MyChart status converted from any "nonactive" status (blank, inactivated, pending activation, patient declined, activation code generated but disabled, and nonstandard MyChart status) to "activated" status within 7 days. Since the beginning of project initiation, active online patient portal status upon hospital discharge increased from a baseline rate of 44 to $66 \%$ (a $22 \%$ absolute increase) within 12 weeks among admitted patients (-Fig. 4).

In this control chart, the upper and lower control limits are set at 3-sigma from the mean to minimize Type I errors, as data points outside of these control limits occur infrequently from common cause variation, and can be used to detect meaningful changes in process performance. Given the process demonstrated a consecutive upward trend of 6 or more data points, this is most consistent with special cause variation, rather than common cause variation, at the end of April 2020. ${ }^{11,12}$ Common cause variation refers to random variation inherent to a specific process that is quantifiable, predictable, and consistent. ${ }^{13,14}$ It is not attributable to specific factor(s) and is not preventable, typically within three standard deviations from the mean or within the control limit. ${ }^{13,14}$ Special cause variation refers to unexpected, nonrandom effects that have not been previously observed. ${ }^{13,14}$ This sporadic variation is caused by specific
Table 3 Demographic comparison between patients who activated and did not activate during their hospitalization in the final week of our study. Basic demographic data of the active and inactive MyChart patient population during the last week of our study

\begin{tabular}{|l|l|l|}
\hline Demographic data & $\begin{array}{l}\text { MyChart } \\
\text { activated } \\
(\boldsymbol{n}=332)\end{array}$ & $\begin{array}{l}\text { MyChart } \\
\text { not activated } \\
(\boldsymbol{n}=155)\end{array}$ \\
\hline Age (mean \pm SD) & $8.2 \pm 5.8$ & $8.2 \pm 6.7$ \\
\hline Gender & $177(53.3 \%)$ & $98(63.2 \%)$ \\
\hline Male & $155(46.7 \%)$ & $57(36.8 \%)$ \\
\hline Female & & \\
\hline Race & $14(4.2 \%)$ & $5(3.2 \%)$ \\
\hline Asian & $17(5.1 \%)$ & $11(7.1 \%)$ \\
\hline Black or African American & $3(0.9 \%)$ & $1(0.6 \%)$ \\
\hline Native- American/Eskimo & $116(34.9 \%)$ & $53(34.2 \%)$ \\
\hline Other & $3(0.9 \%)$ & $1(0.6 \%)$ \\
\hline Other Pacific Islander & $3(0.9 \%)$ & $1(0.6 \%)$ \\
\hline Patient refused & $176(53 \%)$ & $83(53.5 \%)$ \\
\hline White or Caucasian & & \\
\hline Ethnicity & $145(43.7 \%)$ & $83(53.5 \%)$ \\
\hline Hispanic or Latino & $185(55.7 \%)$ & $71(45.8 \%)$ \\
\hline Non-Hispanic & $2(0.6 \%)$ & $1(0.6 \%)$ \\
\hline Patient refused & $297(89.5 \%)$ & $104(67.1 \%)$ \\
\hline Language & $0(0 \%)$ & $4(2.6 \%)$ \\
\hline Arabic & $33(9.9 \%)$ & $46(29.7 \%)$ \\
\hline English & & $1(0.6 \%)$ \\
\hline Other & $3 \%)$ & \\
\hline Spanish & & \\
\hline
\end{tabular}

factor(s) that create a unique change to the process and appear randomly on a control chart, sometimes beyond the control limit or even within the control limit at times. ${ }^{13,14}$ During the same period of time in this study, the percentage of online patient portals converted to active within 7 days increased from 6.0 to $24 \%$. Additionally, the percentage of online patient portal offer rate increased from 73 to $85 \%$ across all inpatient units ( - Fig. 5 ).

\section{Discussion}

We report a dramatic increase in patient portal activation at hospital discharge during a period of high demand for telemedicine as an alternative access to patient care. Our institution found that a multidisciplinary approach to improvement and engagement at multiple levels was successful, starting by addressing barriers to activation and workflow issues. We believe that part of our success was also due to buy-in from unit managers and leaders, leading to a unified effort in making rapid workflow changes within a small window of time. We also continually educated parents and medical staff on the importance of online patient portal 


\section{Inpatient Active MyChart Percentage}

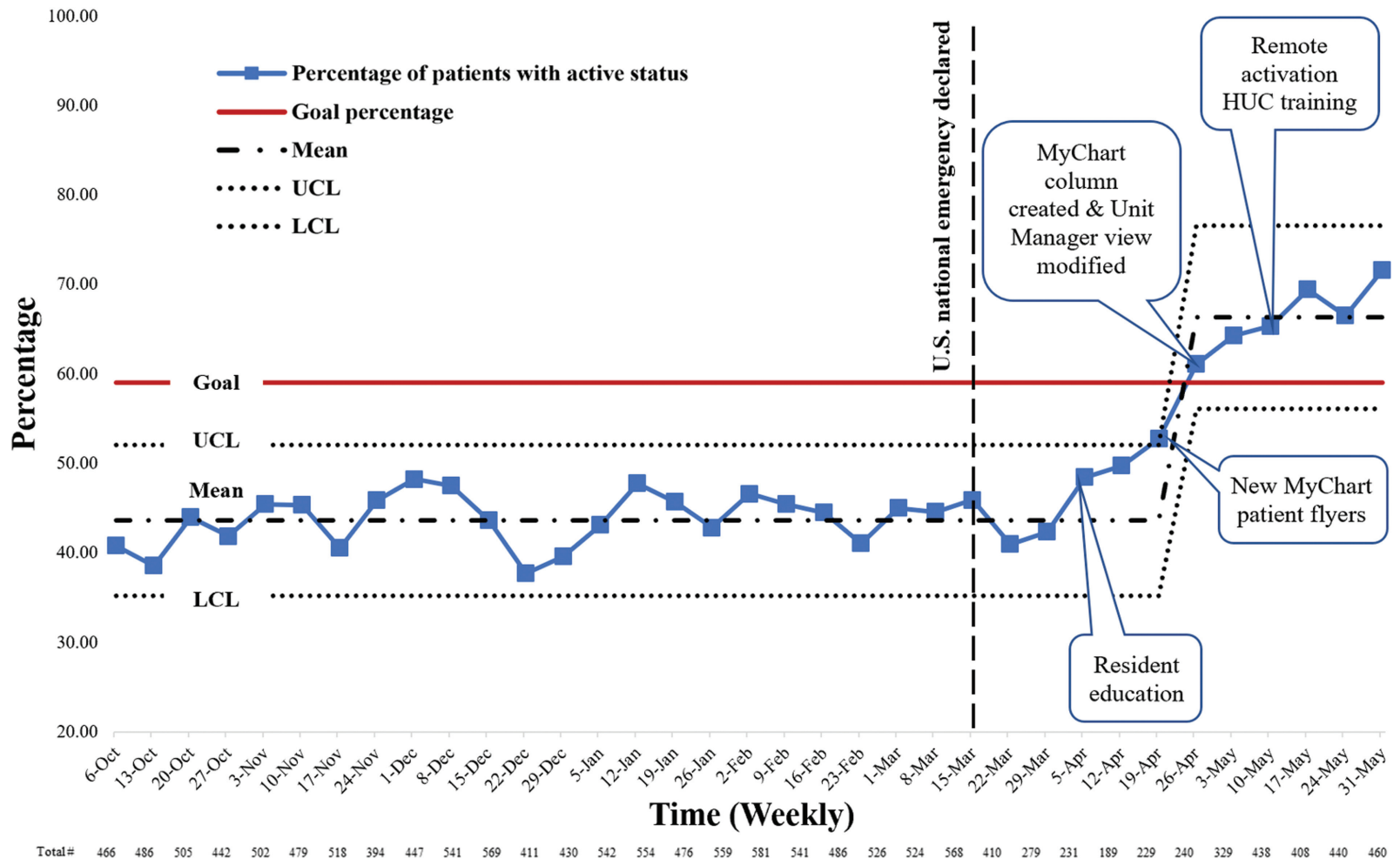

Fig. 4 Percentage of inpatients with active MyChart status upon hospital discharge. Percentage of admitted patients with active online patient portal status upon hospital discharge, ocurring over 12 weeks of our study.

enrollment due to the new physical barriers our community is facing due to COVID-19. While training on the new workflow was limited only to e-mails and tip sheets, members of the task force utilized repetition and follow-up phone calls to enhance our training efforts. Through these efforts, we were gradually promoting a culture change and highlighting the crucial role inpatient staff and providers play in patient portal enrollment. This responsibility was no longer limited to the ambulatory setting.

Another key component to this success was the development of the remote activation workflow. The ability to have an entirely remote proxy enrollment process for families in isolation rooms allowed us to overcome a "physical" barrier while continuing to minimize exposure risk for our staff and medical providers. This remote workflow was further enhanced by two clinical decision support tools. The first was the unit manager view modification, which provides a visual cue of MyChart status, allowing HUCs to drive the new enrollment process. This EMR modification was not custom made; it is an existing feature released by Epic and thus can be easily replicated at other institutions. The second tool utilized was the Epic MyChart status column, which makes portal status visible to medical providers without interfering with their clinical workflows. Utilizing EMR tools to implement a new remote activation workflow, we were able to optimize our efforts in increasing portal enrollment in a relatively short time period.

To date, there is limited literature on patient portal enrollment among pediatric patients. To our knowledge, we are the first to report rapid implementation of a remote online patient/proxy portal enrollment workflow with successful percentage increase in the inpatient pediatric setting. Pediatric online portal enrollment studies have focused mostly on ambulatory settings with activation rates between 30 and $38 \%{ }^{15,16} \mathrm{~A}$ brief report by Patel et al did demonstrate a 10-fold increase in patient portal enrollment in ambulatory clinics when remote enrollment process was implemented. ${ }^{17}$ One of the higher pediatric patient portal enrollment rates was reported by Ramsey et al with a rate of $87.5 \%$; however, the study was done in an adolescent ambulatory setting, which was not comparable to our study due to the significant difference in population and setting. ${ }^{18}$ Adolescent portal access must be differentiated from caregiver proxy access. While we do offer adolescent portal access, our initiative only focused on caregiver proxy enrollment. Other patient portal enrollment studies have mostly been in adults with variable success rates. For example, a systematic review of 40 adult studies (mix of inpatient and outpatient settings) reported an overall mean patient portal adoption rate of $23 \%$ among the 26 studies done in noncontrolled clinical settings. ${ }^{19}$ Another study by Sigler et al reported increased portal enrollment within an adult ambulatory HIV clinic of $6.4 \%$ over a 3-month period $(54.8-61.2 \%) .{ }^{20}$ Currently, the only study focusing on pediatric inpatient enrollment reported an absolute increase in activation from 9 to $26 \% .{ }^{21}$ Our patient portal enrollment percentage is higher than most previously published studies. However, there is a gap in knowledge regarding pediatric and inpatient patient 


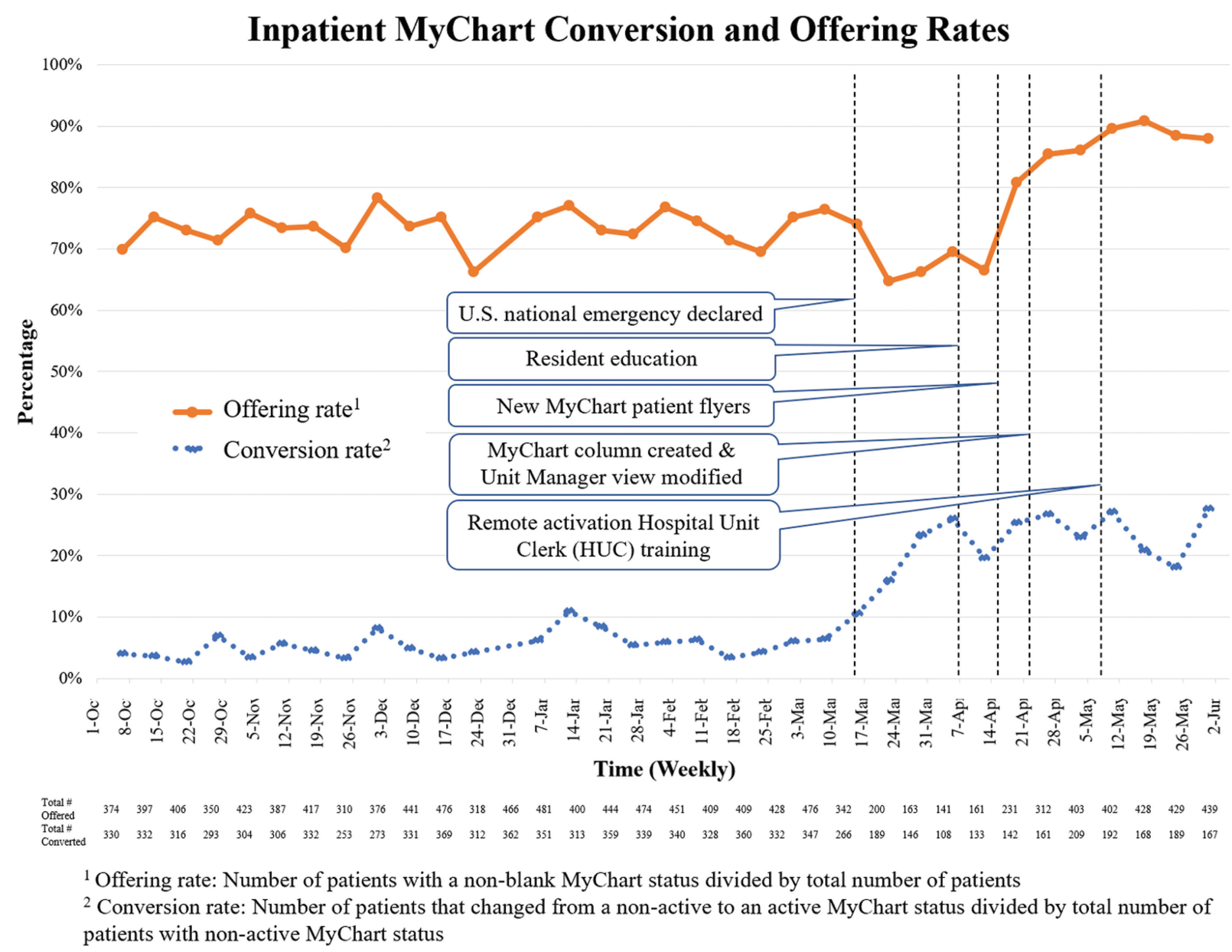

Fig. 5 Inpatient MyChart conversion and offering rates. The percentage of online patient portals converted to active wtihin 7 days after discharge, and the percentage of online patient portal offer rate across all inpatient units.

portal enrollment. While our study addresses this gap, more future research is needed.

Our study identified similar barriers that have been previously reported in the literature. A usability study done by Haggstrom et al demonstrated that a substantial number of users, especially with advanced age, experienced problems with a seemingly straightforward registration process and showed a lack of technical skills. ${ }^{22-24}$ To overcome this barrier, our physicians, nurses, and HUCs have all been trained on MyChart functionalities to assist caregivers in their enrollment. Additionally, our follow-up workflow when caregivers decline enrollment also aims at addressing discomfort with technology and lack of knowledge of patient portal benefits. Emani et al demonstrated that relative advantage was among the top 3 factors, including Ease of Use and Trialability, that lead patients to adopt patient portals. ${ }^{25}$ By using a COVID-specific patient MyChart flyer, we were emphasizing to families a relative advantage of online patient portal over existing practices such as telephoning or visiting the doctor's office. There were, however, some barriers which our study did not address including health literacy, security concerns, race, and socioeconomic status. ${ }^{19,23,24,26,27}$ Further exploration of these barriers and solutions to them are still needed in future steps.
Patient portals promote patient engagement by allowing them to more fully participate in care, allowing patients to view results, notes and medical history, answer questionnaires/surveys, refill prescriptions, schedule appointments, manage co-pays, and communicate securely with the health care team. Despite these benefits, many organizations are challenged with getting patients to enroll. We were no exception. Prior to the pandemic, we had begun an initiative to activate patients in any clinical setting, emphasizing that portal access would allow patients to derive benefit by allowing easy access to notes and results. Progress had been incremental albeit slow; in early March 2020, approximately $50 \%$ of our patients across our entire institution were enrolled in MyChart. The patient portal was viewed as an ambulatory tool with responsibility for activation heavily concentrated in the ambulatory clinic settings. The pandemic transformed our ambulatory practice overnight to telemedicine. Given our integrated video/EMR workflow within the patient portal, we needed to activate patient portal accounts as quickly as possible to allow patients access to telemedicine benefits. Developing a remote activation process for caregiver proxies and focusing all care areas on the benefits of patient portals allowed us to not only increase our enrollment for hospitalized patients, but also for our overall patient population; $72 \%$ of our current 
patients now have active portal accounts. The success of this initiative allows us to engage patients, streamline visits, and improve throughput to meet physical distancing requirements more easily. We believe this experience may be informative for other institutions that aim to increase telehealth visit capabilities and promote online proxy portal enrollment, including pediatric organizations and adult institutions with patients requiring proxies to help manage care. Planned future efforts to address additional identified barriers include patient portal education videos for patients with preferred language of Spanish or Arabic, non-English patient portal application build, and request for additional equipment and personnel resources to help promote portal enrollment.

\section{Limitations}

Despite the success of this initiative, there were some limitations. First, due to COVID-19 physical distancing guidelines, training occurred primarily through e-mails and pictorial tip sheets. The effectiveness of this training is unclear. We would have preferred in-person or live video conferencing which may be a more effective training model for other institutions. Similarly, while a majority of medical patients are cared for by resident physicians, the adequacy and consistency of their training is uncertain, especially given the rotating nature of residents on service and the presence of outside rotators who spend little time on our inpatient units. Second, our study's quality improvement study design limits the ability to determine causality between our intervention and improved MyChart activation, and it is possible that other projects may have influenced our outcomes. There has been a simultaneous effort to increase ambulatory MyChart activation that has been ongoing for several years without noticeable improvement in our inpatient enrollment rate as seen in our consistent baseline rate over 6 months prior to our interventions. Additionally, this initiative was the only project addressing inpatient MyChart activation during this study period and our data only reflect inpatient efforts. While ambulatory effort is unlikely to affect our primary outcome, it could possibly impact the percentage of patients converted to active status within 7 days after discharge if patients were encouraged to enroll or activate their portal account during their posthospitalization follow-up appointment. This could lead to an increase in our conversion rate, although we estimate the effect to be small given posthospitalization appointments were not routinely recommended for all discharged patients during the start of the pandemic. Third, inpatient patient census volume was significantly lower than previous years due to the pandemic. However, this low patient volume was similar to those of other pediatric institutions across the country during a similar time period. It is unclear if our current practices will be sustainable as our patient volume increases, especially during peak respiratory season when a majority of patients will be limited by contact/droplet precautions. Lastly, our study's results are limited by what can be captured by our EMR. Further study will be needed to investigate whether increasing MyChart status rates will increase telehealth visits and utilization of MyChart functionality.

\section{Conclusion}

Our institution is the first to describe a remote patient proxy portal activation workflow that adheres to physical distancing guidelines and which is applicable to all patients requiring proxies to help manage care. We were able to implement this new process successfully and quickly during the COVID19 pandemic. Our rates of online patient portal enrollment improved over the course of several interventions involving both staff education and EMR tools, which are generalizable to other institutions. By increasing online portal enrollment rates, institutions are taking a central step toward engaging patients in, and optimizing access to, care.

\section{Clinical Relevance Statement}

Significant multidisciplinary collaboration and identification of workflow barriers can help improve patient portal activation. Remote activation strategies for proxy enrollment can be effective and allow adherence to physical distancing guidelines, especially when combined with targeted staff education and EMR tools with clinical decision support tools.

\section{Authors' Contributions}

All authors contributed to the writing and editing of this manuscript.

\section{Protections of Human and Animal Subjects}

This project was performed in compliance with the World Medical Association Declaration of Helsinki on Ethical Principles for Medical Research Involving Human Subjects. The project was reviewed by the local institutional review board and was deemed not to be human subjects research. Quality improvement institutional review was obtained and granted.

Funding

None.

Conflict of Interest

None declared.

\section{References}

1 World Health Organization. Pneumonia of unknown cause China. Accessed 2020 at: https://www.who.int/csr/don/05-january-2020-pneumonia-of-unkown-cause-china/en/

2 World Health Organization. Novel Coronavirus - China. Accessed 2020 at: https://www.who.int/csr/don/12-january-2020-novelcoronavirus-china/en/

3 Huang C, Wang Y, Li X, et al. Clinical features of patients infected with 2019 novel coronavirus in Wuhan, China. Lancet 2020;395 (10223):497-506

4 Li Q Guan X, Wu P, et al. Early transmission dynamics in wuhan, china, of novel coronavirus-infected pneumonia. N Engl J Med 2020;382(13):1199-1207

5 Wu Z, McGoogan JM. Characteristics of and important lessons from the coronavirus disease 2019 (COVID-19) outbreak in china: 
summary of a report of 72314 cases from the chinese center for disease control and prevention. JAMA 2020;323(13):1239-1242

6 Holshue ML, DeBolt C, Lindquist S, et al; Washington State 2019nCoV Case Investigation Team. First case of 2019 novel coronavirus in the united states. $\mathrm{n}$ engl $\mathrm{j}$ med 2020;382(10):929-936

7 McMichael TM, Currie DW, Clark S, et al; Public Health-Seattle and King County, EvergreenHealth, and CDC COVID-19 Investigation Team. Epidemiology of COVID-19 in a long-term care facility in King County, Washington. N Engl J Med 2020;382(21): 2005-2011

8 World Health Organization. Director-General's opening remarks at the media briefing on COVID-19 - 11 March 2020. Accessed March 29, 2020 at: https://www.who.int/dg/speeches/detail/ who-director-general-s-opening-remarks-at-the-media-briefing-on-covid-19-11-march-2020

9 Proclamation on declaring a national emergency concerning the novel coronavirus disease (COVID-19) outbreak. Accessed 2020 at: https://www.federalregister.gov/documents/2020/03/18/202005794/declaring-a-national-emergency-concerning-the-novel-coronavirus-disease-covid-19-outbreak

10 Remarks by President Trump, Vice President Pence, and Members of the Coronavirus Task Force in Press Briefing. Accessed April 7, 2020 at: https://www.whitehouse.gov/briefings-statements/remarkspresident-trump-vice-president-pence-members-coronavirustask-force-press-briefing-3/

11 Benneyan JC, Lloyd RC, Plsek PE. Statistical process control as a tool for research and healthcare improvement. Qual Saf Health Care 2003;12(06):458-464

12 Mohammed MA. Using statistical process control to improve the quality of health care. Qual Saf Health Care 2004;13(04):243-245

13 Common-cause versus special-cause. six sigma daily. Published January 4, 2013. Accessed November 7, 2020 at: https://www. sixsigmadaily.com/common-cause-vs-special-cause/

14 Common cause variation versus special cause variation. Simplilearn.com. Published July 28, 2015. Accessed November 7, 2020 at: https://www.simplilearn.com/common-vs-special-cause-ofvariance-article

15 Ketterer T, West DW, Sanders VP, Hossain J, Kondo MC, Sharif I. Correlates of patient portal enrollment and activation in primary care pediatrics. Acad Pediatr 2013;13(03):264-271
16 Ratliff-Schaub K, Valleru J. Increasing patient portal activation in a pediatric subspecialty clinic. Pediatr Qual Saf 2017;2(06):e049

17 Patel PD, Cobb J, Wright D, et al. Rapid development of telehealth capabilities within pediatric patient portal infrastructure for COVID-19 care: barriers, solutions, results. J Am Med Inform Assoc 2020;27(07):1116-1120

18 Ramsey A, Lanzo E, Huston-Paterson H, Tomaszewski K, Trent M. Increasing patient portal usage: preliminary outcomes from the MyChart Genius Project. J Adolesc Health 2018;62(01):29-35

19 Fraccaro P, Vigo M, Balatsoukas P, Buchan IE, Peek N, van der Veer SN. Patient portal adoption rates: a systematic literature review and meta-analysis. Stud Health Technol Inform 2017;245:79-83

20 Sigler R, Kubat R, Lopez A, Newman JR. Implementation of a standardised sign-up process to increase MyChart enrolment among HIV-positive patients. BMJ Open Qual 2019;8(03): e000602

21 Lossius MN, McIntyre D, Resnick W, Hoskavich J. Health system implementation strategies to increase pediatric patient portal use. Pediatrics 2019;144(2 Meeting Abstract):125-125

22 Haggstrom DA, Saleem JJ, Russ AL, Jones J, Russell SA, Chumbler NR. Lessons learned from usability testing of the VA's personal health record. J Am Med Inform Assoc 2011;18(Suppl 1):i13-i17

23 Tieu L, Sarkar U, Schillinger D, et al. Barriers and facilitators to online portal use among patients and caregivers in a safety net health care system: a qualitative study. J Med Internet Res 2015; 17(12):e275

24 Tieu L, Schillinger D, Sarkar U, et al. Online patient websites for electronic health record access among vulnerable populations: portals to nowhere? J Am Med Inform Assoc 2017;24(e1): e47-e54

25 Emani S, Peters E, Desai S, et al. Who adopts a patient portal?: an application of the diffusion of innovation model J Innov Health Inform 2018;25(03):149-157

26 Smith SG, O'Conor R, Aitken W, Curtis LM, Wolf MS, Goel MS. Disparities in registration and use of an online patient portal among older adults: findings from the LitCog cohort. J Am Med Inform Assoc 2015;22(04):888-895

27 Wildenbos GA, Peute L, Jaspers M. Facilitators and Barriers of Electronic Health Record Patient Portal Adoption by Older Adults: A Literature Study. Stud Health Technol Inform 2017;235:308-312 